CUBO A Mathematical Journal

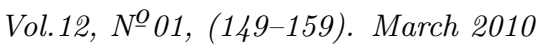

\title{
An Improved Convergence and Complexity Analysis for the Interpolatory Newton Method
}

\author{
IOANNIS K. ARgYROS \\ Cameron University, Department of Mathematical Sciences, \\ Lawton, OK 73505, USA \\ email: iargyros@cameron.edu
}

\begin{abstract}
We provide an improved compared to [5]-[7] local convergence analysis and complexity for the interpolatory Newton method for solving equations in a Banach space setting. The results are obtained using more precise error bounds than before [5]-[7] and the same hypotheses/computational cost.
\end{abstract}

\section{RESUMEN}

Nosotros entregamos aquí un análisis de convergencia local y complejidad para el método de interpolación de Newton para resolver ecuaciones en espacios de Banach. Los resultados mejoran los de [5]-[7] e son obtenidos usando mas precisas cotas de error y las mismas hipotesis y costo computacional.

Key words and phrases: Newton's method, local convergence, Banach space, interpolatory Newton method, complexity, radius of convergence.

Math. Subj. Class.: 65G99, 65H10, 65B05, 47H17, $49 M 15$. 


\section{Introduction}

In this study we are concerned with the problem of approximating a simple solution $\alpha$ of the equation

$$
F(x)=0,
$$

where $F$ is an operator defined on a convex subset $D$ of a Banach space $X$ with values in a Banach space $Y$ over the real or complex fields of dimension $N$,

$$
\operatorname{dim}(X)=\operatorname{dim}(Y)=N, \quad 1 \leq N \leq+\infty .
$$

We consider interpolatory iteration $I_{n}$ for approximating $x^{*}$ defined as follows: Let $x_{i}$ be an approximation to $\alpha$ and let $w_{i}$ be the interpolatory polynomial of degree $\leq n-1$ such that

$$
w_{i}^{(j)}\left(x_{i}\right)=F^{(j)}\left(x_{i}\right), \quad j=0,1, \ldots, n-1 \quad(n \geq 2) .
$$

The next approximation $x_{i+1}^{*}$ is a zero of $w_{i}$. For $n=2$ we obtain Newton's method:

$$
x_{i+1}^{*}=x_{i}-F^{\prime}\left(x_{i}\right)^{-1} F\left(x_{i}\right) \quad(i \geq 0) .
$$

We approximate $x_{i+1}$ by applying a number of Newton iterations to $w_{i}(x)=0$. Let $\left\{x_{i}\right\}$ be the interpolatory Newton iteration $I N_{n}$ given by:

$$
\begin{aligned}
z_{0} & =x_{i} \\
z_{j+1} & =z_{j}-w_{i}^{\prime}\left(z_{j}\right)^{-1} w_{i}\left(z_{j}\right), \quad j=0,1, \ldots, k-1 \\
x_{i+1} & =z_{k}, \quad k=[\log 2 n] .
\end{aligned}
$$

A local convergence analysis and the corresponding complexity of method (1.4) was studied in the elegant paper by Traub and Wozniakowski [7]. Relevant works can be found in [1]-[7], and the references there.

Here we are motivated by paper [7] and optimization considerations. In particular using more precise estimates on the distances $\left\|x_{i}-\alpha\right\|(i \geq 0)$ we show that under the same hypotheses and computational cost as in [5]-[7], we can provide a larger convergence radius, sharper error bounds on the distances and consequently a finer complexity for method (1.4).

Numerical examples are introduced which compare favorably with results to the corresponding ones in [5]-[7].

\section{Local Convergence Analysis of Method (1.4)}

Let $\Gamma \geq 0$. We introduce the closed ball $U=U(\alpha, \Gamma)=\{x \in X \mid\|x-\alpha\| \leq \Gamma\}$, and the parameters

$$
A_{j}=A_{j}(\Gamma)=\sup _{x \in U}\left\|F^{\prime}(\alpha)^{-1} \frac{F^{(j)}(x)}{j !}\right\|, \quad(j \geq 2)
$$


provided that $F^{(j)}$ exists.

Moreover we introduce the parameter $A$ by

$$
A=A(\Gamma)=\sup _{x \in U} \frac{\left\|F^{\prime}(\alpha)^{-1}\left[F^{\prime}(x)-F^{\prime}(\alpha)\right]\right\|}{2\|x-\alpha\|} .
$$

The foundation of our approach and what makes it more precise than the corresponding one in [7] is the fact that we use (2.2) instead of (2.1) (for $j=2$ ) to obtain upper bounds on the crucial quantity $\left\|w_{j}^{\prime}(x)^{-1} F^{\prime}(\alpha)\right\|$.

Indeed, on the one hand note that

$$
A \leq A_{2}
$$

holds in general and $\frac{A_{2}}{A}$ can be arbitrarily large [1], [2]. On the other hand see (2.28), (2.46), and Remark 2.4.

Let us set

$$
a=\frac{A}{A_{2}}, \quad A_{2} \neq 0
$$

Note that $a \in[0,1]$.

We showed in [3] the following improvement of Theorem 2.1 in [6] and Theorem 2.1 in [5] respectively:

Theorem 2.1. If $F$ is twice differentiable in $U$, (2.2) holds and

$$
\begin{gathered}
A_{2} \Gamma \leq \frac{1}{2(1+a)}, \\
x_{i} \in U,
\end{gathered}
$$

then the next approximation $x_{i+1}^{*}$ generated by Newton method (1.3) is well defined, and satisfies for all $i \geq 0$ :

$$
\left\|x_{i+1}^{*}-\alpha\right\| \leq \frac{A_{2}}{1-2 a A_{2}}\left\|x_{i}-\alpha\right\|^{2} \leq \frac{1}{2}\left\|x_{i}-\alpha\right\|
$$

and

$$
x_{i+1}^{*}-\alpha=\frac{1}{2} F^{\prime}(\alpha)^{-1} F^{\prime}(\alpha)\left(x_{i}-\alpha\right)^{2}+O\left(\left\|x_{i}-\alpha\right\|^{2}\right) .
$$

Theorem 2.2. If $F$ is $n$-times differentiable, $n \geq 3$ in $U$, (2.2) holds, and

$$
\begin{gathered}
\frac{n A_{n} \Gamma^{n-1}}{1-a A_{2} \Gamma}<\left(\frac{2}{3}\right)^{n-1} \\
x_{i} \in U,
\end{gathered}
$$

then the polynomial $w_{i}$ has a unique zero in $U^{*}=U^{*}\left(\alpha, \frac{\Gamma}{2}\right)$ and defining $x_{i+1}^{*}$ as the zero of $w_{i}$ in $U^{*}$ the following estimates hold for all $i \geq 0$

$$
\left\|x_{i+1}^{*}-\alpha\right\| \leq \frac{A_{n}\left(1+\left\|x_{i+1}^{*}-\alpha\right\| /\left\|x_{i}-\alpha\right\|\right)^{n}}{1-a A_{2}\left\|x_{i+1}^{*}-\alpha\right\|}\left\|x_{i}-\alpha\right\|^{n} \leq \frac{1}{2}\left\|x_{i}-\alpha\right\|
$$


and

$$
x_{i+1}^{*}-\alpha=\frac{(-1)^{n}}{n !} F^{\prime}(\alpha)^{-1} F^{(n)}(\alpha)\left(x_{i}-\alpha\right)^{n}+O\left(\left\|x_{i}-\alpha\right\|^{n}\right) .
$$

We can show the main local convergence theorem for method (1.4):

Theorem 2.3. If $F$ is $n$-times differentiable, $n \geq 3$ in $U$, (2.2) holds, and

$$
0 \leq \tilde{A}_{2} \Gamma \leq \frac{1}{3+2 a}
$$

where,

$$
\begin{gathered}
\tilde{A}_{2}=\frac{A_{2}+\frac{n(n-1)}{2} A_{n}(2 \Gamma)^{n-2}}{1-a A_{2} \Gamma-n A_{n}\left(\frac{3}{2}\right)^{n-1} \Gamma^{n-1}} \\
x_{0} \in U,
\end{gathered}
$$

then sequence $\left\{x_{i}\right\}(i \geq 0)$ generated by interpolary-Newton iteration $I N_{n}$ is well defined, remains in $U$ for all $i \geq 0$, converges to $\alpha$ so that the following estimates hold for all $i \geq 0$ :

$$
\begin{aligned}
e_{i+1}=\left\|x_{i+1}-\alpha\right\| & \leq\left\{\frac{1}{2}+\frac{3}{2}\left(\frac{1}{2}\right)^{k}\right\} e_{i}, \\
e_{i+1} & \leq c_{i, n} e_{i}^{n}
\end{aligned}
$$

where,

$$
\begin{aligned}
c_{i, n}= & \left(1+\frac{e_{i+1}^{*}}{e_{i}}\right)\left[\frac{A_{n}}{1-a A_{2} e_{i+1}^{*}}\right. \\
& \left.+\left(\tilde{A}_{2}\left(1+H_{i}\right)\right)^{2^{k}-1}\right]\left(\left(1+\frac{e_{i+1}^{*}}{e_{i}}\right) e_{i}\right)^{2^{k}-n}
\end{aligned}
$$

for

$$
\begin{gathered}
e_{i+1}^{*}=\left\|x_{i+1}^{*}-\alpha\right\|, H_{i}=O\left(e_{i}\right), 0 \leq H_{i} \leq \frac{3+2 a}{2}, k=[\log 2 n], \\
\lim _{i \rightarrow \infty} c_{i, n}=A_{n}+\delta \tilde{A}_{2}^{n-1} \text { where } \delta=0 \\
\quad \text { if } 2^{k}>n \text { and } \delta=1, \text { if } 2^{k}=n, \\
x_{i+1}-\alpha=F_{n}\left(x_{i}-\alpha\right)^{n}+b_{i, k}+O\left(\left\|x_{i}-\alpha\right\|^{n}\right),
\end{gathered}
$$

where

$$
\begin{aligned}
b_{i, 1} & =F_{2}\left(x_{i}-\alpha\right)^{2}, \\
b_{i, j+1} & =F_{2} b_{i, j}^{2}, \quad j=1,2, \ldots, k-1,
\end{aligned}
$$

and

$$
F_{j}=\frac{(-1)^{j}}{j !} F^{\prime}(\alpha)^{-1} F^{(j)}(\alpha) \text { for } j=2 \text { and } n .
$$


The proof is similar to Theorem 3.1 in [7], but there are differences where we use (2.2) instead of (2.1) (for $i=2$ ).

Proof. We shall first show using induction on $j \geq 0$ that $w_{j}^{\prime}\left(z_{j}\right)$ is invertible and $z_{j} \in U$.

Set

$$
F^{(j)}(x)-w_{i}^{(j)}(x)=R_{n}^{(j)}\left(x ; x_{i}\right), \quad x \in U, j=0,1,2,
$$

where,

$$
\left\|F^{\prime}(\alpha)^{-1} R_{n}^{(j)}\left(x ; x_{i}\right)\right\| \leq j !\left(\begin{array}{c}
n \\
j
\end{array}\right) A_{n}\left\|x-x_{i}\right\|^{n-1} .
$$

We can write

$$
\begin{aligned}
w_{i}^{\prime}(x) & =F^{\prime}(x)-R_{n}^{\prime}\left(x ; x_{i}\right) \\
& =F^{\prime}(\alpha)\left[I+F^{\prime}(\alpha)^{-1}\left\{F^{\prime}(x)-F^{\prime}(\alpha)\right\}-F^{\prime}(\alpha)^{-1} R_{n}^{\prime}\left(x ; x_{i}\right)\right]
\end{aligned}
$$

and in view of (2.2), (2.12) and (2.24) for $x \in U$ we get in turn

$$
\begin{aligned}
\left\|F^{\prime}(\alpha)^{-1}\left[w_{j}^{\prime}(x)-F^{\prime}(\alpha)\right]\right\| & \leq 2 a A_{2}\|x-\alpha\|+n A_{n}\left\|x-x_{i}\right\|^{n-1} \\
& \leq 2 a A_{2} \Gamma+n A_{n}(2 \Gamma)^{n-1} \leq \frac{2}{3+2 a}<1 .
\end{aligned}
$$

It follows from (2.28) and the Banach Lemma on invertible operators [4] that $w_{i}^{\prime}(x)$ is invertible for all $x \in U$, and

$$
\left\|w_{i}^{\prime}(x)^{-1} F^{\prime}(\alpha)\right\| \leq \frac{1}{1-2 a A_{2}\|x-\alpha\|-n A_{n}\left\|x-x_{i}\right\|^{n-1}} .
$$

Since the denominator in $(2.13)$ is positive we get

$$
\frac{n A_{n} \Gamma^{n-1}}{1-a A_{2} \Gamma}<\left(\frac{2}{3}\right)^{n-1}
$$

and from Theorem $2.2 w_{i}$ has a unique zero $x_{i+1}^{*}$ in $U^{*}$ and (2.10) holds.

Using (2.24) and (2.29) we get for $x \in U$

$$
\begin{aligned}
\left\|w_{j}^{\prime}\left(x_{i+1}^{*}\right)^{-1} \frac{w_{i}^{\prime \prime}(x)}{2}\right\| & \\
& \leq\left\|w_{i}^{\prime}\left(x_{i+1}^{*}\right)^{-1} F^{\prime}(\alpha)\right\|\left\|F^{\prime}(\alpha)^{-1} \frac{w_{i}^{\prime \prime}(x)}{2}\right\| \\
& \leq \frac{A_{2}+\frac{n(n-1)}{2} A_{n}\left\|x-x_{i}\right\|^{n-2}}{1-2 a A_{2}\left\|x_{i+1}^{*}-\alpha\right\|-n A_{n}\left\|x_{i+1}^{*}-x_{i}\right\|^{n-1}} \\
& \leq \frac{A_{2}+\frac{n(n-1)}{2} A_{n}(2 \Gamma)^{n-2}}{1-a A_{2} \Gamma-n A_{n}\left(\frac{3}{2} \Gamma\right)^{n-1}}=\tilde{A}_{2} .
\end{aligned}
$$

It follows from Theorem 3.1 and (2.12) that for $z_{1}=x_{i}-F^{\prime}\left(x_{i}\right)^{-1} F\left(x_{i}\right)$

$$
\left\|z_{1}-\alpha\right\| \leq \frac{1}{2}\left\|x_{i}-\alpha\right\| .
$$


Since $x_{i+1}^{*} \in U^{*},\left\|z_{1}-x_{i+1}^{*}\right\| \leq \Gamma$, we shall show

$$
z_{j+1} \in D_{j}=\left\{x:\left\|x-x_{i+1}^{*}\right\| \leq \frac{1}{2}\left\|z_{j}-x_{i+1}^{*}\right\|\right\} \cap U .
$$

Set

$$
w_{i}(x)=w_{i}\left(z_{j}\right)+w_{i}^{\prime}\left(z_{j}\right)\left(x-z_{j}\right)+\bar{R}_{2}\left(x ; z_{j}\right),
$$

where,

$$
\bar{R}_{2}(x ; y)=\int_{0}^{1} w_{i}^{\prime \prime}(y+t(x-y))(x-y)^{2}(1-t) d t .
$$

Note that $z_{j+1}$ is the solution of equation

$$
x=H(x)=x_{i+1}^{*}+w^{\prime}\left(x_{i+1}\right)^{-1}\left\{\bar{R}_{2}\left(x ; z_{j}\right)-\bar{R}_{2}\left(x ; x_{i+1}^{*}\right)\right\} .
$$

We shall show $H$ is contractive on $D_{j}$.

It follows from (2.12), (2.31) and (2.36):

$$
\begin{aligned}
\left\|H(x)-x_{i+1}^{*}\right\| & \leq \tilde{A}_{2}\left(\left\|x-z_{j}\right\|^{2}+\left\|x-x_{i+1}^{*}\right\|^{2}\right) \\
& \leq \frac{2+3 a}{2} \tilde{A}_{2}\left\|z_{j}-x_{i+1}^{*}\right\| \leq \frac{1}{2}\left\|z_{j}-x_{i+1}^{*}\right\| .
\end{aligned}
$$

Moreover we have

$$
\|H(x)-\alpha\| \leq\left\|x_{i+1}^{*}-\alpha\right\|+\left\|H(x)-x_{i+1}^{*}\right\| \leq\left(\frac{1}{2}+\frac{1}{2}\right) \Gamma=\Gamma .
$$

It follows by the contraction mapping principle [4], (2.37) and (2.38) that $z_{j+1}$ is the unique zero of $H$ in $D_{j}$. It follows that $x_{i+1}=z_{k} \in U$, and

$$
\begin{aligned}
\left\|x_{i+1}-\alpha\right\| & \leq\left\|x_{i+1}-x_{i+1}^{*}\right\|+\left\|x_{i+1}^{*}-\alpha\right\| \\
& \leq\left(\frac{1}{2}\right)^{k}\left\|z_{0}-x_{i+1}^{*}\right\|+\frac{1}{2}\left\|x_{i}-\alpha\right\| \\
& \leq\left[\frac{3}{2}\left(\frac{1}{2}\right)^{k}+\frac{1}{2}\right]\left\|x_{i}-\alpha\right\| \leq \frac{7}{8}\left\|x_{i}-\alpha\right\|,
\end{aligned}
$$

which shows $x_{i} \in U$ and (2.15) hold true.

Set $\bar{e}_{j}=\left\|z_{j}-x_{i+1}^{*}\right\|$ and $x=z_{j+1}$ in (2.36). Then we get

$$
\bar{e}_{j+1} \leq \frac{\tilde{A}_{2}\left(1+\frac{\bar{e}_{j+1}}{\bar{e}_{j}}\right)^{2}}{1-\tilde{A}_{2} \bar{e}_{j+1}} \bar{e}_{j}^{2} \leq \tilde{A}_{2}\left(1+H_{i}\right) \bar{e}_{j},
$$

where $H_{i}=O\left(\bar{e}_{j}\right)$ and $0 \leq H_{i} \leq \frac{2+3 a}{2}$ compare to (2.7). In view of $\bar{e}_{j}=O\left(e_{j}\right)$ we can set 
$H_{i}=O\left(e_{i}\right)$. It follows from $(2.10)$ and $(2.40)$

$$
\begin{aligned}
e_{i+1} \leq & \left\|x_{i+1}-x_{i+1}^{*}\right\|+\left\|x_{i+1}^{*}-\alpha\right\|=\bar{e}_{k}+\left\|x_{i+1}^{*}-\alpha\right\| \\
\leq & {\left[\tilde{A}_{2}\left(1+H_{i}\right)\right]^{2^{k}-1}\left\|x_{i}-x_{i+1}^{*}\right\|^{2^{k}} } \\
& +\frac{A_{n}}{1-a A_{2} e_{i+1}^{*}}\left(1+\frac{e_{i+1}^{*}}{e_{i}}\right)^{n} e_{i}^{n} \\
\leq & \left(1+\frac{e_{i+1}^{*}}{e_{i}}\right)^{n}\left(\frac{A_{n}}{1-a A_{2} e_{i+1}^{*}}\right. \\
& \left.+\left[\tilde{A}_{2}\left(1+H_{i}\right)\right]^{2^{k}-1}\left[\left(1+\frac{e_{j+1}^{*}}{e_{i}}\right) e_{i}\right]^{2^{k}-n}\right) e_{i}^{n}=c_{i, n} e_{i}^{n} .
\end{aligned}
$$

In view of $\frac{e_{i+1}^{*}}{e_{i}}$ and $H_{i}$ tending to zero we get

$$
\lim _{i \rightarrow \infty} c_{i, n}=A_{n}+\delta \tilde{A}_{2}^{n-1},
$$

where $\delta=0$ if $2^{k}>n$ and $\delta=1$ otherwise. Hence, (2.16) holds.

Furthermore, we have

$$
\begin{aligned}
z_{j+1}-x_{i+1}^{*} & =w_{i}^{\prime}\left(x_{i+1}^{*}\right)^{-1} \frac{w_{i}^{\prime \prime}\left(x_{i+1}^{*}\right)}{2}\left(z_{j}-x_{i+1}^{*}\right)^{2}+O\left(\tilde{e}_{j}^{3}\right) \\
& =F^{\prime}(\alpha)^{-1} \frac{F^{\prime \prime}(\alpha)}{2}\left(z_{j}-x_{i+1}^{*}\right)^{2}+O\left(e_{i+1}^{*} \tilde{e}_{j}^{2}+\tilde{e}_{j}^{3}\right) \\
& =F_{2}\left(z_{j}-x_{i+1}^{*}\right)^{2}+O\left(\tilde{e}_{j}^{2}\right) .
\end{aligned}
$$

Therefore, we get

$$
\begin{aligned}
z_{k}-x_{i+1}^{*} & =F_{2}\left(F_{2} \cdots\left(F_{2}\left(x_{i}-x_{i+1}^{*}\right)^{2}\right)^{2} \cdots\right)^{2}+O\left(e_{i}^{2 k}\right) \\
& =F_{2}\left(F_{2} \cdots\left(F_{2}\left(x_{i}-\alpha\right)^{2}\right)^{2} \cdots\right)^{2}+O\left(e_{i}^{2 k}\right) .
\end{aligned}
$$

In view of (2.21), (2.22), and (2.44) we have

$$
z_{k}-x_{i+1}^{*}=b_{i, k}+O\left(e_{i}^{2 k}\right) .
$$

In view of (2.11) and (2.45) we deduce

$$
x_{i+1}-\alpha=z_{k}-x_{i+1}^{*}+x_{i+1}^{*}-\alpha=b_{i, k}+F_{n}\left(x_{i}-\alpha\right)^{n}+O\left(e_{i}^{n}\right),
$$

which shows (2.20).

That completes the proof of the theorem.

Remark 2.4. The less precise estimate (using (2.1) for $j=2$ instead of sharper (2.2) that is actually needed)

$$
\left\|F^{\prime}(\alpha)^{-1}\left[w_{j}^{\prime}(x)-F^{\prime}(\alpha)\right]\right\| \leq 2 A_{2}\|x-\alpha\|+n A_{n}\left\|x-x_{i}\right\|^{n-1}
$$


was used in [7] instead of (2.28), together with

$$
0 \leq \tilde{A}_{2} \Gamma \leq \frac{1}{5}
$$

instead of weaker (2.12).

If $A=A_{2}$ our results Theorem 2.1, Theorem 2.2 and Theorem 2.3 reduce to the corresponding Theorem 2.1 in [6], Theorem 2.1 in [5] and Theorem 3.1 in [7] respectively. Otherwise our results constitute improvements with advantages already stated in the Introduction.

We now give conditions under which $I N_{n}$ enjoys a "type of global convergence".

Let

$$
F(x)=\sum_{i=1}^{\infty} \frac{1}{\iota !} F^{(i)}\left(x_{i}-\alpha\right)^{i}
$$

be analytic in $D=U^{0}(\alpha, R)$, and

$$
\frac{\left\|F^{\prime}(\alpha)^{-1} F^{(i)}(\alpha)\right\|}{\iota !} \leq K^{i-1}
$$

for $i \geq 2$ and $R \geq \frac{1}{K}$.

As in [7], one way to find $K$ is to use Cauchy's formula

$$
\frac{\left\|F^{\prime}(\alpha)^{-1} F^{(i)}(\alpha)\right\|}{\iota !} \leq \frac{M}{R^{i}},
$$

where,

$$
M=\sup _{x \in D}\left\|F^{\prime}(\alpha)^{-1} F(x)\right\| .
$$

Let $K=\max \left[\frac{1}{R}, \frac{M}{R^{2}}\right]$. Then

$$
\frac{M}{R} \leq K R \leq(K R)^{i-1}
$$

and

$$
\frac{M}{R^{i}} \leq K^{i-1}
$$

We can show:

Theorem 2.5. If (2.2) and (2.50) hold then the interpolary Newton method (1.4) converges provided that $x_{0} \in U\left(\alpha, \Gamma_{n}\right)$, where

$$
\Gamma_{n}=\frac{x_{n}}{K}
$$

and $x_{n}, 0<x_{n}<x_{\infty}$, satisfies the equation

$$
\begin{aligned}
& (3+2 a)\left[\frac{x}{(1-x)^{3}}+\frac{n(n-1)}{4(1-x)^{2}}\left(\frac{2 x}{1-x}\right)^{n-1}\right] \\
& \quad=1-\frac{a x}{(1-x)^{3}}-\frac{n}{(1-x)^{2}}\left[\frac{3 x}{2(1-x)}\right]^{n-1}
\end{aligned}
$$


and $x_{n} \rightarrow x_{\infty}$, where

$$
x_{\infty} \geq .12
$$

is the positive solution of equation

$$
\frac{x}{(1-x)^{3}}=\frac{1}{4+2 a}
$$

Proof. In view of (2.50) we have for

$$
f(x)=\frac{x}{1-K x}
$$

that

$$
\left\|F^{\prime}(\alpha)^{-1} F^{(i)}(x)\right\| \leq f^{(i)}(\|x-\alpha\|) .
$$

Using

$$
f^{(i)}(x)=\frac{i ! K^{i-1}}{(1-K x)^{i+1}} \quad(i \geq 2)
$$

we get

$$
A_{i}(\Gamma) \leq \frac{K^{i-1}}{(1-K \Gamma)^{i+1}} \quad(i \geq 2) .
$$

It follows from (2.13) and (2.62) that

$$
\tilde{A}_{2} \Gamma \leq \frac{\left[\frac{K \Gamma}{(1-K \Gamma)^{3}}+\frac{n(n-1)}{4(1-K \Gamma)^{2}}\left(\frac{2 K \Gamma}{1-K \Gamma}\right)^{n-1}\right]}{1-\frac{a K \Gamma}{(1-K \Gamma)^{3}}-\frac{n}{(1-K \Gamma)^{2}}\left(\frac{3 K \Gamma}{2(1-K \Gamma)}\right)^{n-1}}=\frac{1}{3+2 a} .
$$

Letting $K \Gamma=x$ we see that $x$ satisfies equation (2.56). It is simple calculus to show that $x=x(n)$ is an increasing function of $n$ and $x_{\infty}=\lim _{n \rightarrow \infty} x(n)$ satisfies equaiton (2.58).

Remark 2.6. If $A=A_{2}$ (i.e. $a=1$ ) our Theorem 2.5 reduces to Theorem 3.2 in [7]. Otherwise it is an improvement, since the limit of sequence $x(n)$ in [7] is .12 which is smaller than ours implying by $(2.55)$ that we provide a larger radius of convergence.

In particular if $R$ is related to $\frac{1}{K}$, say $R=\frac{c_{1}}{K}$, then

$$
\Gamma_{n}=\frac{x_{n}}{K}=\frac{x_{n}}{c_{1} R} \leq \frac{x_{\infty}}{c_{1} R} .
$$

The rest of the results introduced in [7] are improved. In particular with the notation introduced in [7] we have for

$$
\begin{aligned}
& \text { I: } e_{i}=G_{i} e_{i-1}^{n}, G_{i} \leq \bar{G}, \\
& \qquad \bar{G}=\bar{G}(n)= \begin{cases}\frac{A_{2}}{1-2 a A_{2} \Gamma}, & n=2 \\
(1+q)^{n}\left[\frac{A_{n}}{1-a A_{2} \frac{\Gamma}{2}}+\left(\frac{7}{2} \tilde{A}_{2}\right)^{2^{k}-1}[(1+q) \Gamma]^{2^{k}-n},\right. & n>2\end{cases}
\end{aligned}
$$


where $\tilde{A}_{2}$ is given by $(2.13), q=\frac{1}{2}+\frac{3}{2}\left(\frac{1}{2}\right)^{k}$, and $K=[\log 2 n]$.

II: If the total number of arithmetic operations necessary to solve a system of $N$ linear equations is $O\left(n^{\beta}\right), \beta \leq 3$, then

$$
d\left(I N_{n}\right)= \begin{cases}O\left(N^{\beta}[\log 2 n]+N^{2}\left(\begin{array}{c}
N+n-2 \\
n-2
\end{array}\right)([\log 2 n]-1)\right. & \text { for } N \geq 2, \\
(3+2 a)[\log 2 n]+O(1), & \text { for } n=1 .\end{cases}
$$

Remark 2.7. If $A=A_{2}$ our results reduce to the ones in [7]. Otherwise they constitute an improvement.

We complete this study with an example to show that strict inequality can hold in (2.3):

Example 2.8. Let $X=Y=\mathbf{R}, x^{*}=0$ and define function $F$ on $U=U(0,1)$ by

$$
F(x)=e^{x}-1 .
$$

Using (2.1), (2.2), (2.4) and (2.66) we obtain

$$
A=\frac{e-1}{2}<\frac{e}{2}=A_{2}
$$

and

$$
a=.632120588 .
$$

It follows from (2.5) that our radius of convergence is given by

$$
\Gamma_{A}=.112699836 .
$$

The corresponding radius $\Gamma_{T W}$ given in Theorem 2.1 in $[6]$ or $[7]$ is:

$$
\Gamma_{T W}=\frac{1}{4 A_{2}}=.09196986 .
$$

That is

$$
\Gamma_{T W}<\Gamma_{A}
$$

Received: October, 2008. Revised: January, 2009.

\section{References}

[1] Argyros, I.K., A unifying local-semilocal convergence analysis and applications for twopoint Newton-like methods in Banach space, J. Math. Anal. Applic., 298 (2004), 374-397. 
[2] Argrros, I.K., Approximate Solution of Operator Equations with Applications, World Scientific Publ. Comp., River Edge, New Jersey, 2005.

[3] Argyros, I.K., An improved convergence and complexity analysis of Newton's method for solving equations, (to appear).

[4] Kantorovich, L.V. And Akilov, G.P., Functional Analysis in Normed Spaces, Moscow, 1959.

[5] Ortega, J.M. and Rheinboldt, W.C., Iterative Solution of Nonlinear Equations in Several Variables, Academic Press, New York, 1970.

[6] Traub, J.F. and Wozniakowski, H., Strict lower and upper bounds on iterative computational complexity. In Analytic Computational Complexity, J.F. Traube, Ed., Academic Press, New York, 1976, pp. 15-34.

[7] Traub, J.F. and Wozniakowski, H., Convergence and complexity of Newton iteration for operator equations, J. Assoc. Comput. Machinery, 26, No. 2 (1979), 250-258. 